\title{
Quarkonium Formation at High Energy
}

\author{
R. L. Thews ${ }^{\text {a* }}$

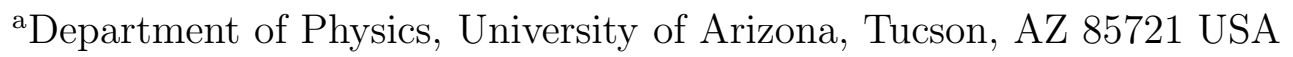

The production of quarkonium in heavy ion collisions is studied at RHIC and LHC energies. General arguments indicate that, due to initial production of multiple quark pairs in each central collision, the final population of quarkonium may exhibit significant enhancements over straightforward extrapolation of behavior at SPS energy. Explicit calculations based on both a statistical hadronization picture and a kinetic formation mechanism in a deconfined state verify these general expectations. Such enhancements will alter the nature of how quarkonium yields may be used as a signature of deconfinement.

\section{GENERIC EXPECTATIONS FOR LARGE $N_{c \bar{c}}$}

At heavy ion collision energies at RHIC and LHC, the initial number of heavy quark pairs produced in each collision will be qualitatively different than the number produced at SPS energies. For example, the number of charm quark pairs is expected to be of the order of ten at RHIC and several hundred at LHC in the most central collisions [1]. Let us attempt to extract features of $J / \psi$ formation from the initially-produced charm quarks which are independent of detailed dynamics. We consider scenarios in which the formation of $J / \psi$ is allowed to proceed through any combination of one of the $N_{c}$ charm quarks with one of the $N_{\bar{c}}$ anticharm quarks which result from the initial production of $N_{c \bar{c}}$ pairs in a central heavy ion collision. For a given charm quark, one expects then that the probability $\mathcal{P}$ to form a $J / \psi$ is just proportional to the number of available anticharm quarks relative to the number of light antiquarks,

$$
\mathcal{P} \propto N_{\bar{c}} / N_{\bar{u}, \bar{d}, \bar{s}} \approx N_{c \bar{c}} / N_{c h},
$$

where we normalize the number of light antiquarks by the number of produced charged hadrons. Since this ratio is generally very small, one can simply multiply by the number of available charm quarks $N_{c}$ to obtain the total number of $J / \psi$ expected in a given event.

$$
N_{J / \psi} \propto N_{c \bar{c}}^{2} / N_{c h},
$$

where the use of the initial values $N_{c \bar{c}}=N_{c}=N_{\bar{c}}$ is again justified by the relatively small number of bound states formed. For an ensemble of events, the average number of $J / \psi$ per event is calculated from the average value of initial charm $\left\langle N_{c \bar{c}}\right\rangle$, and we neglect fluctuations in $N_{c h}$.

$$
<J / \psi>=\lambda\left(<N_{c \bar{c}}>+1\right)<N_{c \bar{c}}>/ N_{c h},
$$

${ }^{*}$ Supported by U. S. Department of Energy Grant DE-FG03-95ER40937 
where we place all dynamical dependence in the parameter $\lambda$. [One can extend this formula to the case where $J / \psi$ formation is effective not over the entire rapidity range $Y_{\text {total }}$, but only if the quark and antiquark are within the same rapidity interval $\Delta y$. In this case one makes the replacement $\left\langle N_{c \bar{c}}>+1 \rightarrow<N_{c \bar{c}}>+Y_{\text {total }} / \Delta y\right.$.]

The essential property of this result is that the growth with energy of the quadratic dependence on total charm [1] is expected to be much stronger than the corresponding growth of total particle production in heavy ion collisions [2]. $J / \psi$ production without this quadratic mechanism is typically some small energy-independent fraction of total initial charm production [3], so that we can expect the quadratic formation to become dominant at high energy.

We show numerical results in Table 1 for these quantities with a prefactor $\lambda$ of unity. Estimates for the charm and particle numbers are very approximate, but serve to show the anticipated trend with energy. At SPS, this formation mechanism is most probably insignificant. At RHIC it is comparable with "normal" formation, while at LHC one might expect it to be dominant. Of course, the exact result will depend on the details of the physics which controls the formation.

Table 1

Comparison of $J / \psi$ formation variation with energy

\begin{tabular}{llll}
\hline & $S P S$ & RHIC & LHC \\
\hline$\sqrt{s}(\mathrm{GeV})$ & 18 & 200 & 5500 \\
$\left\langle N_{c \bar{c}}\right\rangle$ & 0.2 & 10 & 200 \\
$N_{c h}$ & 1350 & 3250 & 16500 \\
$\left\langle N_{J / \psi}\right\rangle$ & 0.00018 & 0.034 & 2.4 \\
$N_{J / \psi}^{\text {initial }}$ & 0.0012 & 0.06 & 1.2 \\
\hline
\end{tabular}

\section{STATISTICAL HADRONIZATION MODEL}

This model is motivated by the success of attempts to explain the relative abundances of light hadrons produced in high energy interactions in terms of the predictions of a hadron gas in chemical and thermal equilibrium [4]. Such fits, however, are not able to describe the abundances of hadrons containing charm quarks. This can be understood in terms of the long time scales required to approach chemical equilibrium for heavy quarks. However, it is expected that for high energy heavy ion collisions the initial production of charm quark pairs exceeds the number expected at chemical equilibrium as determined by the light hadron abundances. The statistical hadronization model [5] assumes that at hadronization these charm quarks are distributed into hadrons according to chemical equilibrium, but adjusted by a factor $\gamma_{c}$ which accounts for oversaturation of charm. One power of this factor multiplies a given thermal hadron population for each charm or anticharm quark contained in the hadron. Thus the relative abundance of $J / \psi$ to that of $\mathrm{D}$ mesons, for example, will be enhanced in this model. The enhancement factor 
is determined by conservation of charm, again using the time scale argument to justify neglecting pair production or annihilation before hadronization.

$$
N_{c \bar{c}}=\frac{1}{2} \gamma_{c} N_{\text {open }} \frac{I_{1}\left(\gamma_{c} N_{\text {open }}\right)}{I_{0}\left(\gamma_{c} N_{\text {open }}\right)}+\gamma_{c}^{2} N_{\text {hidden }},
$$

where $N_{\text {open }}$ and $N_{\text {hidden }}$ are calculated in the thermal equilibrium model grand canonical ensemble. The ratio of Bessel functions is an approximate correction factor [6] which converts to canonical ensemble particle numbers. This factor will only be significant for non-central collisions or lower energies in which the absolute number of charm pairs is quite small [7].

We show in Figure 1 calculated $\gamma_{c}$ values. At large total charm there is linear behavior, which leads to the quadratic growth of $J / \psi$ formation. It is interesting to note that the canonical suppression effect can be very well approximated by using a grand canonical formalism, supplemented by the averaging process for $N_{c \bar{c}}^{2}$ over a distribution of events.

Figure 2 shows several applications for RHIC conditions. The centrality dependence is modeled by the number of nucleon participants, and one sees the change in shape due to the transition between canonical and grand canonical formalism. The absolute magnitudes of $\langle J / \psi>/$ charm are comparable with the initial production estimates of a fraction of a percent, indicating that this process may overwhelm suppression for central collisions at RHIC.

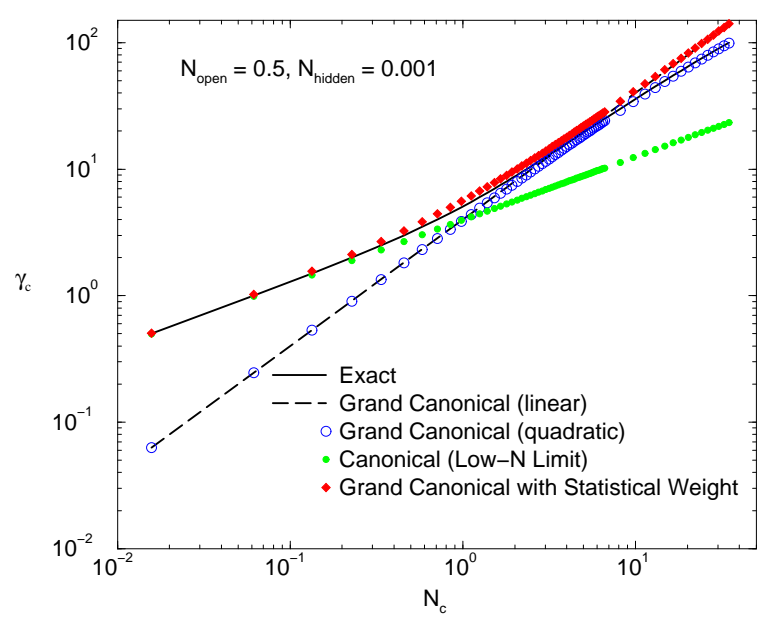

Figure 1. Relation between charm enhancement factor and total number of charm quarks for statistical hadronization model.

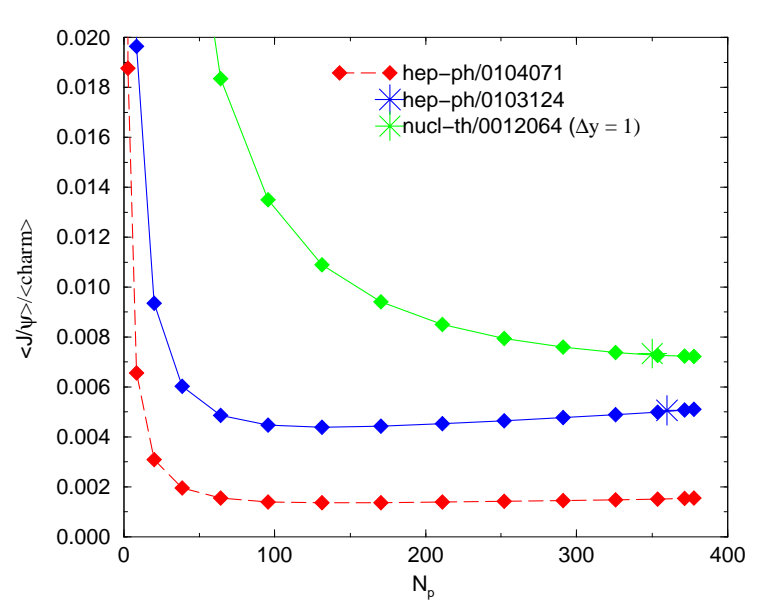

Figure 2. Ratio $<J / \psi>$ over initial charm at RHIC for several applications of the statistical hadronization model.

\section{KINETIC FORMATION MODEL}

In this model, we investigate the possibility to form $J / \psi$ directly in a deconfined medium. The formation will take advantage of the mobility of initially-produced charm 
quarks in a spatial region of deconfinement, such that all combinations of a charm plus anticharm are allowed to form a bound state. For the purposes of this study, we consider a physical picture of deconfinement in which quarkonium is suppressed via collisions with free gluons [8]. The dominant formation process, in which a quark and an antiquark in a relative color octet state are captured into a color singlet bound quarkonium state and emit a color octet gluon, is simply the inverse of the breakup reaction. It is then an inevitable consequence of this picture of suppression that the corresponding formation process must also take place.

The time evolution if the $J / \psi$ population is given by the rate equation

$$
\frac{d N_{J / \psi}}{d \tau}=\lambda_{\mathrm{F}} N_{c} N_{\bar{c}}[V(\tau)]^{-1}-\lambda_{\mathrm{D}} N_{J / \psi} \rho_{g},
$$

with $\rho_{g}$ the number density of gluons. The reactivity $\lambda$ is the reaction rate $\left\langle\sigma v_{\text {rel }}\right\rangle$ averaged over the momentum distribution of the initial participants, i.e. $c$ and $\bar{c}$ for $\lambda_{F}$ and $J / \psi$ and $g$ for $\lambda_{D}$. The gluon density is determined by the equilibrium value in the QGP at each temperature. The system undergoes a longitudinal isentropic expansion, which fixes the time-dependence of the volume $\mathrm{V}(\tau)$. It is evident that the solution of Equation 5 grows quadratically with initial charm $N_{c \bar{c}}$, as long as the total $J / \psi \ll N_{c \bar{c}}$.

$$
N_{J / \psi}\left(\tau_{f}\right)=\epsilon\left(\tau_{f}\right) \times\left[N_{J / \psi}\left(\tau_{0}\right)+N_{c \bar{c}}^{2} \int_{\tau_{0}}^{\tau_{f}} \lambda_{\mathrm{F}}[V(\tau) \epsilon(\tau)]^{-1} d \tau\right]
$$

where $\tau_{f}$ is the hadronization time determined by the initial temperature $\left(T_{0}\right.$ is a variable parameter) and final temperature $\left(T_{f}=150 \mathrm{MeV}\right.$ ends the deconfining phase). The function $\epsilon\left(\tau_{f}\right)=e^{-\int_{\tau_{0}}^{\tau_{f}} \lambda_{\mathrm{D}} \rho_{g} d \tau}$ would be the suppression factor in this scenario if the formation mechanism were neglected.

The momentum distribution of the charm quarks is allowed to vary over a wide range of possibilities. At one extreme we use a thermal equilibrium distribution at the QGP temperature. We also consider a distribution unchanged from that introduced in the initial perturbative QCD processes, plus several intermediate distributions with decreasing rapidity widths. The centrality dependence can also be modeled, using nuclear collision geometry to set the various spatial scales. For details, see Ref. [9,10].

We show the resulting ratios $N_{J / \psi} / N_{c \bar{c}}$ in Figure 3 (for RHIC) and Figure 4 (for LHC) for a full range of the initial charm quark rapidity distributions. Shown for comparison are some statistical hadronization model results, plus an indication of the value if only initial (vacuum) production were present. It is evident that although the absolute values depend strongly on the charm momentum distribution, the magnitudes are substantially above that expected if only initial production plus plasma suppression were present. The centrality dependence is quite striking. Even after normalizing to initial charm, the ratio rises with increasing centrality.

\section{SUMMARY}

Expectations based on general grounds for enhanced formation of heavy quarkonium in relativistic heavy ion collisions have been verified in two different models. In particular, one expects at RHIC and LHC to see an enhancement in the heavy quarkonium formation 


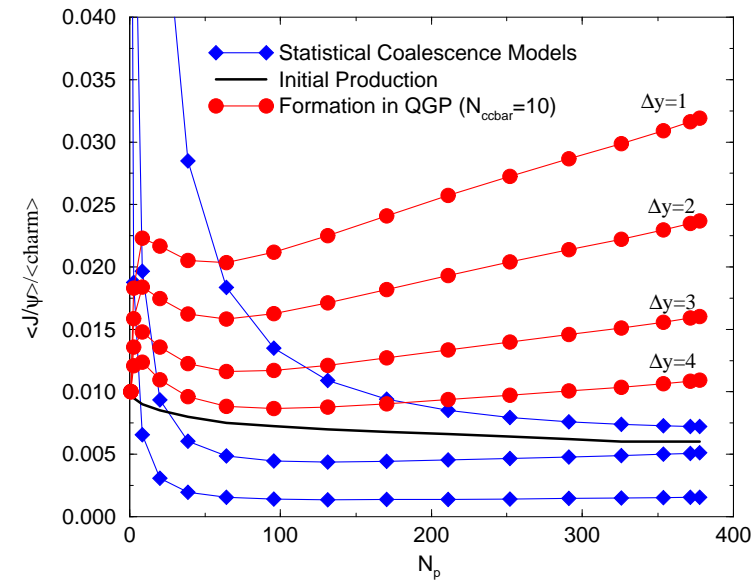

Figure 3. Predictions of kinetic model for $<J / \psi>$ over initial charm at RHIC energy.

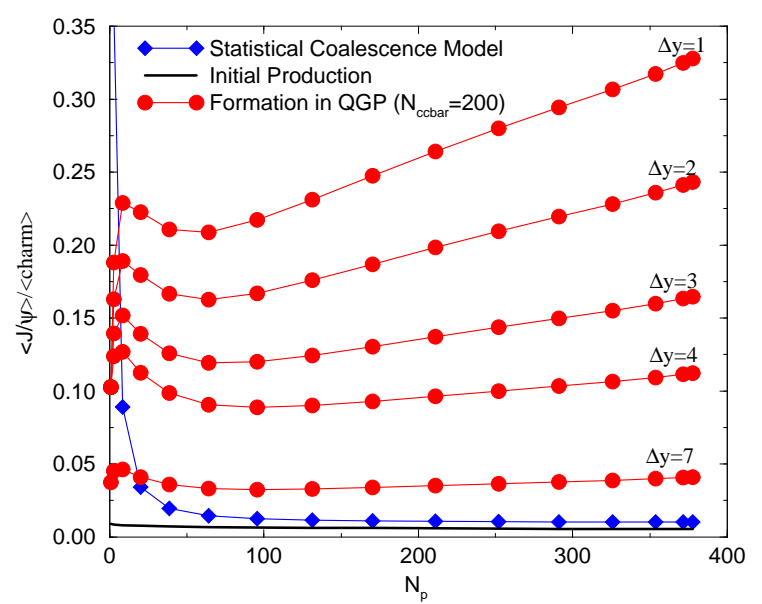

Figure 4. Predictions of kinetic model for $<J / \psi>$ over initial charm at LHC energy.

rate, even when compared to unsuppressed production via elementary nucleon-nucleon collisions in vacuum. The magnitude of this effect is expected to grow with the centrality of the heavy ion collision, just opposite to the predictions of various suppression scenarios. The physics bases for these models, however, are quite distinct. Their differences should manifest themselves in details of the magnitudes and centrality dependence. In this regard, it is essential to have a simultaneous measurement of open flavor production to serve as an unambiguous baseline.

\section{REFERENCES}

1. P. L. McGaughey, E. Quack, P. V. Ruuskanen, R. Vogt, and X.-N. Wang, in "Hard Processes in Hadronic Interactions", Int. J. Mod. Phys. A10 (1995) 2999.

2. X.-N. Wang and M. Gyulassy, Phys. Rev. Lett. 86 (2001) 3496.

3. R. Gavai, D. Kharzeev, H. Satz, G. Schuler, K. Sridhar, and R. Vogt, in "Hard Processes in Hadronic Interactions", Int. J. Mod. Phys. A10 (1995)3043.

4. See for example P. Braun-Munzinger, D. Magestro, K. Redlich, and J. Stachel, Phys. Lett. B518 (2001) 41, and references therein.

5. P. Braun-Munzinger and J. Stachel, Phys. Lett. B490 (2000) 196.

6. J. Cleymans, K. Redlich, and E. Suhonen Z. Phys. C51 (1991) 137.

7. M.I. Gorenstein, A.P. Kostyuk, H. Stocker, and W. Greiner, Phys. Lett. B509 (2001) 277; P. Braun-Munzinger and J. Stachel, Nucl. Phys. A690 (2001) 119.

8. D. Kharzeev and H. Satz, Phys. Lett. B334 (1994) 155.

9. R. L. Thews, M. Schroedter, and J. Rafelski, Phys. Rev. C63 (2001) 054905.

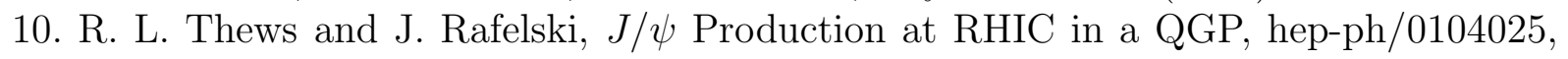
to be published in proceedings of QM2001. 